Positive Electrification due to Heating Aluminium Phosphate

This content has been downloaded from IOPscience. Please scroll down to see the full text. 1909 Proc. Phys. Soc. London 22410

(http://iopscience.iop.org/1478-7814/22/1/329)

View the table of contents for this issue, or go to the journal homepage for more

Download details:

IP Address: 129.93.16.3

This content was downloaded on 10/09/2015 at 18:54

Please note that terms and conditions apply. 


\section{Positive Electrification due to Heating Aluminium} Phosphate. By A. E. GARRETT, B.Sc.*

[Plate VI.]

I. Introduction and Experimental Arrangements.

IN a paper published in the 'Philosophical Magazine' for October, 1904, by Dr. R. S. Willows and myself, it was found that the halogen compounds of zinc when heated are able to discharge both positively and negatively electrified bodies. A more detailed examination of this phenomenon was subsequently carried out by one of us $\dagger$.

In those experiments the temperature to which the salts were raised was in no case higher than $360^{\circ} \mathrm{C}$., and no series of observations at pressures lower than a few $\mathrm{mm}$. were undertaken.

Sir J. J. Thomson $\ddagger$ made some experiments to determine whether the base or the acid is instrumental in producing the ionization, and came to the conclusion that the nature of the jonic charge is determined by the acid. Thus he found that phosphates when heated produce a very large excess of positive ions, halogen compounds produce an excess of positive ions, and nitrates an excess of positive at first, but when heated sufficiently to be converted into oxides they produce an excess of negative. Incidentally he found that aluminium phosphate gives off a very large excess of positive ions.

Now the halogen and other compounds used in the previous experiments are known to be bodies which undergo decomposition when strongly heated, and the ionization in this case may be due to chemical action. Aluminium phosphate, on the other hand, is an extremely stable substance, and it is of interest to investigate the source of ionization in this instance. This salt was therefore chosen for detailed examination.

* Read June 10, 1910.

+ Garrett, Phil. Mag., June 1907.

$\ddagger$ Cambridge Phil. Soc. Proc., p. 105, 1907. 
GAIRETT.
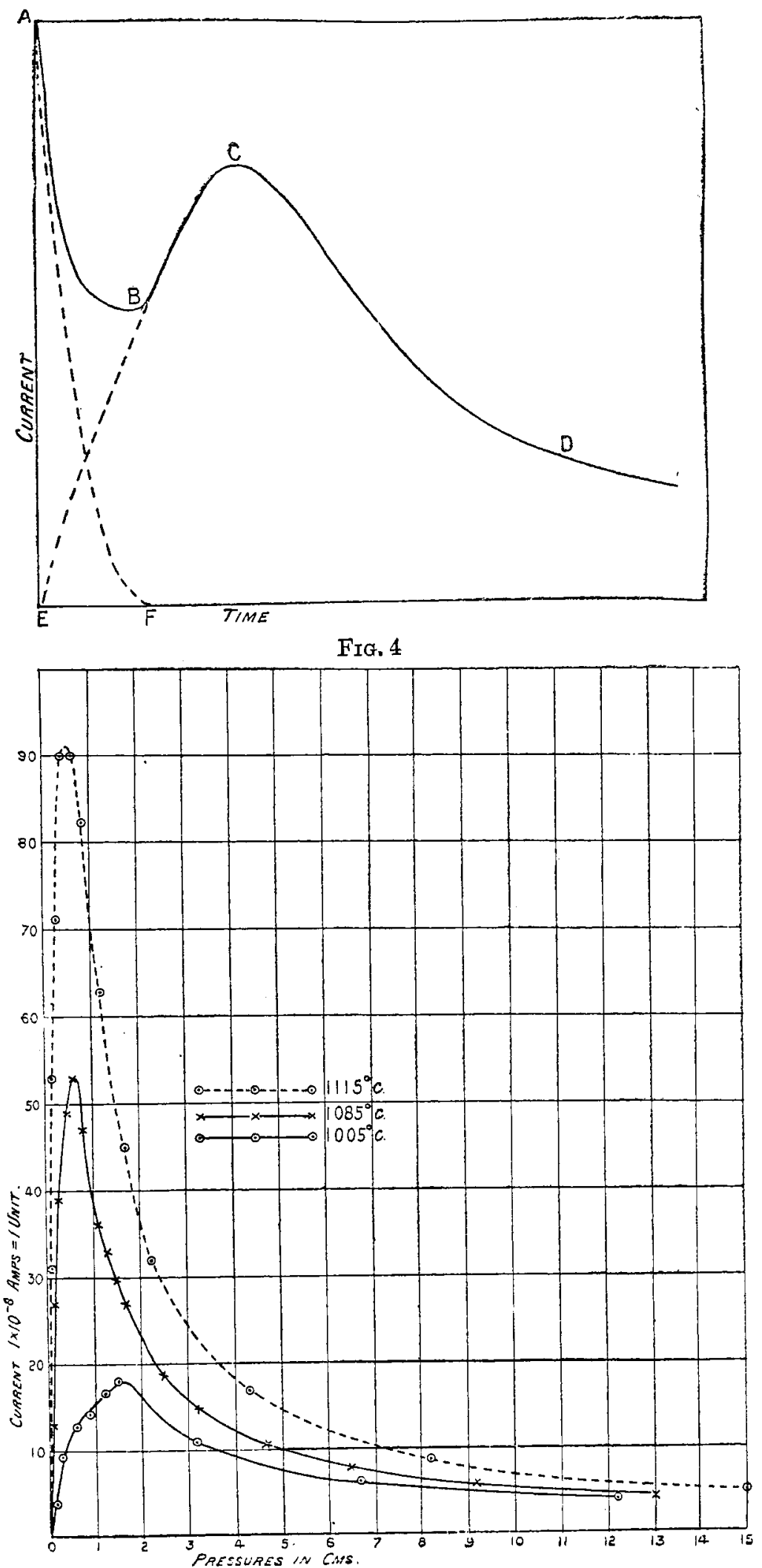

Proc. Plyss. Soc. Vol. XXL1., Pl.VI.

FIG. 3.

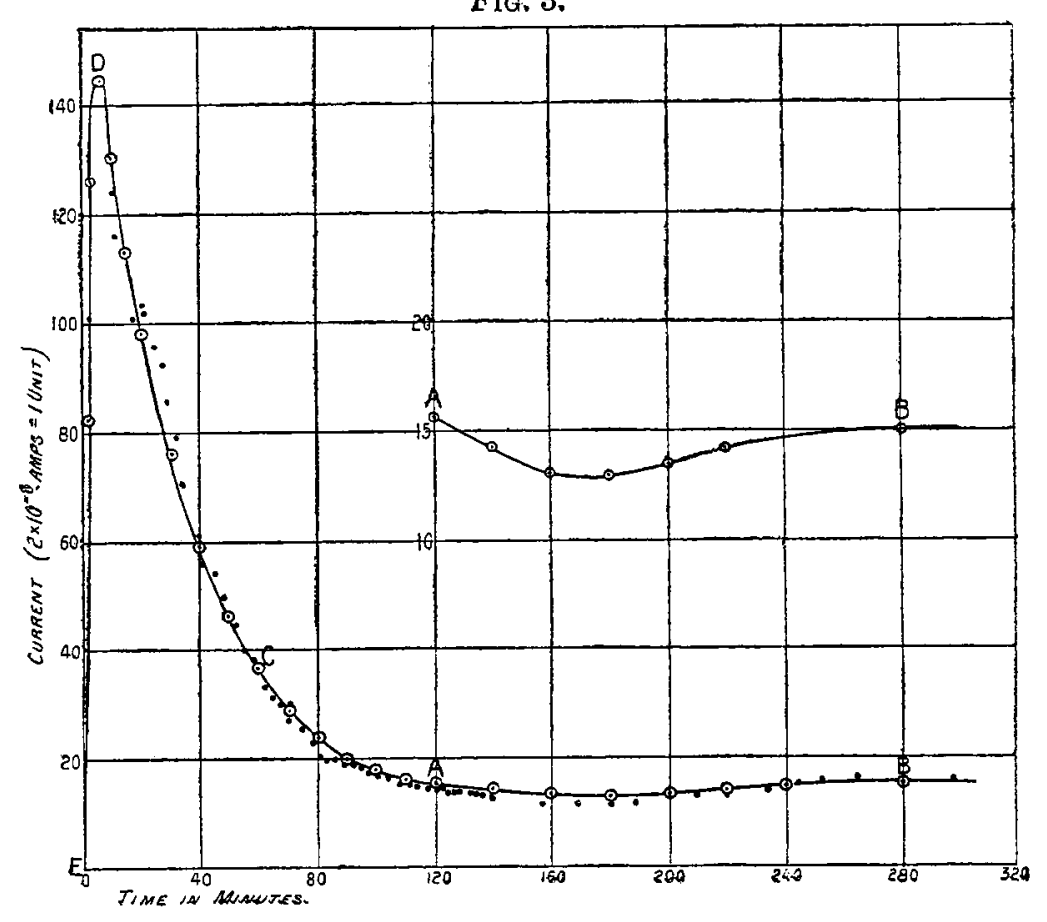

FIG. 5.

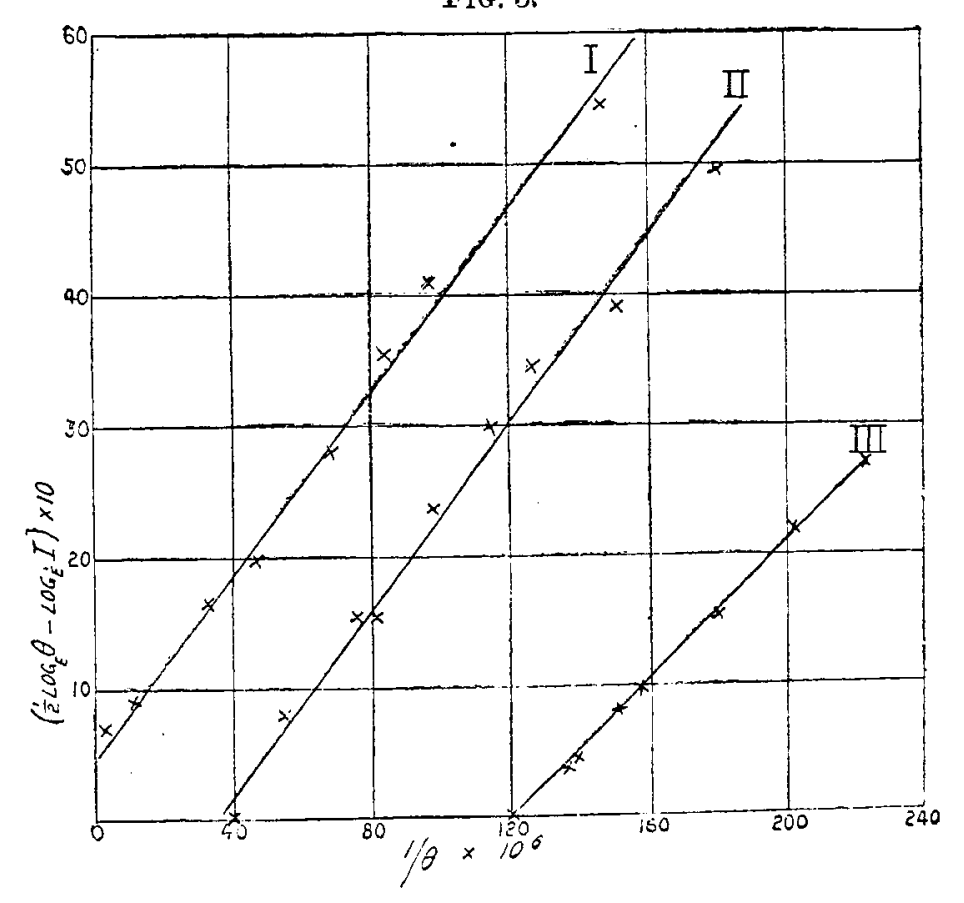


The apparatus used is shown in the following diagram.

The glass tube consists of two parts connected by a groundglass joint. The part $A$ is shown in vertical section, the part $B$ in horizontal section. $e$ is the strip of thin platinum foil, $\frac{1}{4}$ sq. $\mathrm{cm}$. in area, on which the salt is placed to be heated; the temperature of the platinum $e$ is raised by a current, the leads for which are the thick copper wires $c, d$. The electrode $\mathrm{E}$, which is connected through a galvanometer to earth, is an

Fig. 1.

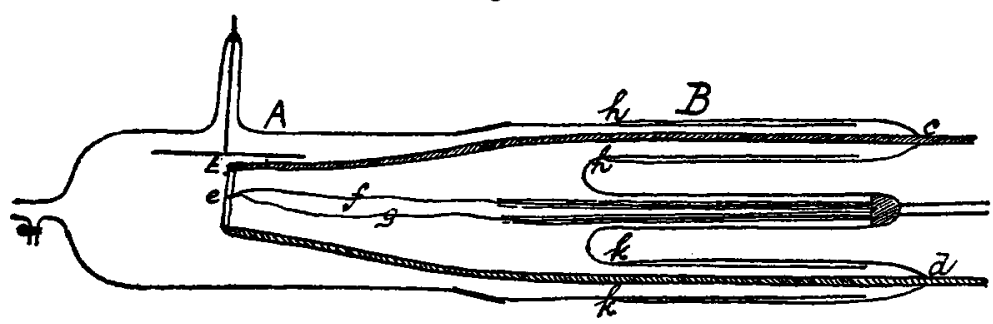

oblong aluminium disk about $3 \mathrm{sq}$. $\mathrm{cm}$. in area; the distance between the heated salt and this electrode was in most cases $0.5 \mathrm{~cm}$. $f, g$ are wires of the thermo-electric couple used for ascertaining the temperature of the platinum foil with which they are fused. To obtain the temperature from the thermoelectric current, the deflexion of the galvanometer to which leads from the junction pass was noted when a tiny particle of $\mathrm{K}_{2} \mathrm{SO}_{4}$ just melted on the foil. In this way the deflexion for two temperatures differing by about $1000^{\circ} \mathrm{C}$. was obtained, and the temperature in degrees centigrade corresponding to any other deflexion could be got by aid of the correction curves given by Callendar *. The reliability of this method was checked by observing the melting-point of $\mathrm{Na}_{2} \mathrm{SO}_{4}$. The observations of the behaviour of aluminium phosphate were taken over a range of temperature from $900^{\circ} \mathrm{C}$. to $1300^{\circ} \mathrm{C}$.

Before commencing work with aluminium phosphate it was ascertained that only a small current due to ions of either sign could be detected when the platinum foil was used alone.

* Phil, Mag. [5] xlviii, pp. 519 et seq.

Yor. XXII. 
The sensitivity of the galvanometer was such that a deflexion of one scale division represented a current of $2 \times 10^{-9}$ ainpere.

When taking readings, a saturation voltage obtained from a battery of small accumulators the negative terminal of which was earthed, was put on $e$ (fig. 1) as soon as the heating of the salt was commenced, and the deflexion of the galvanometer noted from time to time. When the voltage was taken off $e$ in order to read the thermo-current, time was allowed, when the voltage was again put on, for the current to become steady before readings were taken.

When the heating-current had been continued for some time, such a large amount of heat had been conducted along the copper leads as to cause the melting of the wax which was used to render the tube air-tight. This was remedied by soldering a small metal tube over each lead as shown in fig. 1 , and making air-tight wax joints at $h$ and $k$.

During the course of the experiments in which the galvanometer was used, many attempts under varying conditions were made to detect the presence of negative ions, but with no success. That such ions are present was afterwards proved, but from the results obtained they must be less than $\frac{1}{2}$ per cent. of the positive.

Now if the positive ions are produced by chemical change brought about by the heat, then one would expect that a decay in the current would take place with the time. It was found that the current does decrease when heating is continued, so it was decided to obtain the curve of decay for this substance.

\section{Ilecay of Positive Tonization.}

The aluminium phosphate was made into a paste with distilled water and then placed on the foil $e$; the foil was heated slightly by the current before putting it in the tube, this causes the phosphate to adhere to the platinum, and also gets rid of the excess of water. To lessen the effect of the contained gas the tube was evacuated, and the temperature of the foil quickly raised to the degree desired. Varying conditions of temperature and pressure were tested, and it was found that the most expedien.t nethod was to reduce the 
pressure to about $0.05 \mathrm{~cm}$., and to use a temperature of about $1200^{\circ} \mathrm{C}$. Under such circumstances it was possible to obtain a record of the decay in 5 or 6 hours.

The method of procedure was as follows : -+60 volts were put on $e$ (fig. 1), and as soon as the temperature became steady, readings were taken ; the pressure and temperature were kept constant throughout.

Experiments of this kind were carried out with air, hydrogen, and $\mathrm{CO}_{2}$, as the gas in the tube. It was found in every case when air or hydrogen was used, that the manner in which the current varied with the time for the first 20 minutes was peculiar.

A typical curve to illustrate this is given (Pl. VI. fig. 2). In this curve the currents are plotted as ordinates and the times as abscissæ.

This shows a rapid fall from $A$ to $B$, then a rise to a maximum at $\mathrm{C}$, and lastly a decay $\mathrm{C}$ to $\mathrm{D}$, in which the current decreases roughly exponentially with the time. The part of the curve near B sometimes showed still further irregularities.

With $\mathrm{CO}_{2}$ as the gas in the tube, a typical decay curve is represented by ECD. The initial changes observed in air, and hydrogen, are apparently due to water, since a preliminary heating of the phosphate at a lower temperature sufficient to expel the water, gets rid of them altogether.

Further, in the case of $\mathrm{CO}_{2}$, that gas may possibly assist in the removal of hygroscopic moisture, and so prevent its action on the salt.

After some hours' heating, a more or less steady state was reached. This state persisted for some months, nor did it regain any activity if dry or moist air was admitted, even if left for 2 or 3 days. Only on one occasion was a slight temporary regain noted, and in this case the interval was 16 days.

If after the steady state was reached the salt was moistened with distilled water, there was a large increase in the current which quickly died away, and in about 10 minutes the steady state was again reached. The decay of this, AF (Pl. VI. fig. 2), being taken in conjunction with a typical decay curve ECD, a curve of the form ABCD is obtained. 


\section{MR. A. E. GARRETT ON POSITIYE ELECTRIFICATION}

This is furthor evidence that the initial changes are due to water. These changes will not be further considered.

A typical decay-curve over a longer period omitting these changes is shown in Pl. VI. fig. 3.

The part of the curve near $C$ is exponential ; the portion EDC is very similar to the curve given by Rutherford in the 2nd edition of 'Radioactivity,' p. 342, for the variation in activity of the active deposit of Th due to a very short exposure to the emanation. In the case of Rutherford's curve, this is known to be due to the decay of two substances, Th A and $T h B$, the former of which does not produce any rays, and the equation of the curve is of the form $\mathrm{A}\left(e^{-\lambda_{1} t}-e^{-\lambda_{g} t}\right)$. The curve of decay of aluminium phosphate can be represented by an equation of the same form up to the point $\mathrm{C}$, beyond this the exponential curve lies below the experimental. Neglecting this latter point for the moment, if the physical analogy as well as the algebraical one holds, we can suppose the first effect of the high temperature is to produce from the phosphate and the surrounding gas a substance $A$ which does not emit ions, that this next produces a substance $B$ which is the agent producing the conductivity. As in Rutherford's case, the curve alone does not allow one to say to which substance the different $\lambda$ 's refer.

If the analogy holds further and the salt also independently produces $\mathrm{C}$, the latter supplying ions but decaying so slowly that its decay can be neglected, the activity due to $\mathrm{C}$ is represented by a curve having an equation of the form $\mathrm{B}\left(1-e^{-\lambda_{s} t}\right)$, and the whole curve EDCAB should be capable of being fitted by an equation

$$
\mathrm{A}\left(e^{-\lambda_{2} t}-e^{-\lambda_{2} t}\right)+\mathrm{B}\left(1-e^{-\lambda_{3} t}\right) \text {. }
$$

This is actually found to be the case. The values of the $\lambda$ 's depend of course upon the temperature of the salt.

In Pl. VI. fig. 3 the dots represent experimental points, the $\odot$ 's points calculated from the above formula.

It can be seen from the curve, that immediately after the very rapid decay there is a somewhat steady state which is in turn succeeded by a gradual rise in the current to a final steady state. Owing to the small scale used, this is not well shown in the curve at $A B$, although in the experiment there 
illustrated the actual rise amounted to about 20 per cent. of the previous steady values. The upper curve $A B$ represents this section on a larger scale and makes this point more distinct.

The relatively large current at the beginning of the heating appears to depend very largely upon the nature of the gas in the tube, while the final steady current is due entirely to the heated salt.

The results obtained with hydrogen were always of a most irregular nature. The rate of decay was also much slower in that gas than in air or $\mathrm{CO}_{2}$. It was quicker in $\mathrm{CO}_{2}$ than in air.

No alteration of the gas contained makes any difference to the current when in the steady condition if the pressure and temperature are kept constant. Further, although an increased current is obtained with higher temperatures, and an alteration in the pressure produces also an alteration in the current, yet on bringing both temperature and pressure to their former values, the same steady current is obtained.

\section{Attempts to increase the Activity.}

Richardson* found that a platinum wire which is heated to such a temperature that an excess of positive ions are given off, gradually becomes less and less active in this respect until a more or less steady state is reached. When the wire reaches this state he found that it could be rendered much more active by passing, for about a minute, an electric discharge through the tube containing the wire, and he further showed that this regained activity persisted for a considerable time after the discharge had ceased.

It was thought that a discharge might have a similar effect upon the heated phosphate when it had reached the steady state. The heating-current was therefore cut off and a discharge from a Ruhmkorff coil sent through the tube. This increased the current temporarily. If the coil was again applied less effect was produced, until, after several repetitions, the coil produced no effect.

The actual period during which the discharge lasted was

* Phil. Mag. [6] pp. 83 et seq., 1903 . 
gradually increased, and it was found that the maximum effect was attained when the discharge continued for $\frac{1}{2}$ minute. On no occasion did it require more than 20 minutes again to reach the original steady state after discontinuing the discharge. In order to ascertain whether the temporary increase in current was due to the effect which the discharge produced upon the salt itself, or upon the residual gas, a fresh supply of $\mathrm{CO}_{2}$ was admitted when the state at which the coil discharge produced no effect was reached, and the tube pumped down to $0.12 \mathrm{~cm}$. pressure. The coil discharge again caused a temporary increase. The direction of the coil discharge did not influence the result.

The extra current therefore appears to be due to an effect produced by the action of the discharge upon the surrounding gas.

\section{Action of Heat in the alsence of Electrostatic Field.}

When the electrode $e$ was insulated while the heating wis continued, an abnormally large current was obtained so soon as the field was put on becween eand $\mathrm{E}$ (fig. 1). This current gradually decayed, but it was some 2 or 3 minutes before it reached its normal ralue. The magnitude of the increased current was found to depend upon the time during which $e$ was insulated. It gradually increased with the time, and reached a maximum when the insulation had lasted ten minutes. Any longer period of insulation was found to have no further increasing effect upon the current.

It is possible that during the time of insulation positive ions are being freed in the salt on ' $e$,' these accumulite in the substance and are prevented from escaping by a discontinuity of the potential at the surface. This continues until, at the end of 10 minutes, the field due to the accumulated charges is sufficient to take them over as they are formed, when of course no further increase in the number accumulated tilsis place.

When the field is again put on, these ions are of course dragged out, and the initial value of the increased current will depent upon the number of ions which have accumulated.

This effect greatly increased the labour of taking readings 
under varying conditions, as considerable time had to the allowed after a change had been made for the current to become steady. These experiments were carried out after the steady state had been reached.

This effect was more apparent in some gases than in others, of those tried it was most marked in the case of $\mathrm{CO}_{2}$.

\section{Effect of Pressure upon Current.}

When the salt is in the steady state it is most convenient for investigating the changes due to alterations of pressure and temperature.

The changes in the current when the temperature was kept constant while the pressure was varied were first undertaken. The contained gases used were air and $\mathrm{CO}_{2}$. The results obtained were of a similar nature for both these gases.

Some typical curves are shown in the accompanying diagram (Pl. VI. fig. 4), in which the pressures are plotted as abscissæ, and the currents as ordinates.

From these it can be seen that starting from zero pressure there is a very rapid rise in the current in all cases, the higher the temperature the more rapid being the rise. For each temperature the current reaches a well-marked maximum value. This, again, is more pronounced at the higher temperatures. When the maximum current has been obtained, any further increase in the pressure produces an immediate and rapid decrease in the current. For the temperatures investigated, this decrease continues until the pressure attains the value 5 or $6 \mathrm{cms}$, after this the decrease which takes place in the current for any further increase in the pressure is always smaller but is still quite marked. The rate of decrease is always more rapid at the higher temperatures.

When the pressures for which the currents have maximum values are plotted against the temperatures, it appears as if the pressure and temperature are connected by a straight-line law, and that if the temperature could be pushed to a sufficiently high degree the largest current would be obtained in the highest attainable vacuum. 


\section{YR. A. E. GARTELT ON POSITIVE FIJCTRTHCATION}

The fact that the maximum currents obtained at the higher temperatures and lower pressures were always greater than those obtained with the lower temperatures and higher pressures is further evidence in support of this view.

It is quite possible, lowever, that the slope of the temperature-pressure line may gradually become less and less, and that it never actually reaches the zero pressure line.

The peculiar manner in which the current varies with the pressure under these conditions cannot be explained by the collision of moving ions, as in all cases the voltage used was that corresponding to the flat part of the saturation curve. Fvidence given later suggests that neutral douhlets, such as Righi suggested compose the magneto-cathode rays, and Sir J. J. Thomson * found indications of in his experiments on positive electricity, are driven off when the salt is heated, and the current may be due to the break up of these doublets by collision with the gas molecules.

These doublets would be shot off with relatively large velocities at the lower pressures, but would have smill chances of colliding with gas molecules or other doublets, and so, relatively few free ions are produced. At higher pressures the doublets would have much slower speeds but greater chauces of collision. Somewhere between these extremes the most favourable conditions for obtaining a maximum current may be looked for at each temperature.

At the higher temperatures the velocity of ejection is greater, and a less number of collisions will be required in order to split up the doublet, hence the maximum current is obtained under such conditions at a lower pressure.

With an increase of pressure more collisions are possible, but less doublets escape from the heated salt since the temperature is lower, hence the current obtained is smaller.

'The great drawback in the way of this explanation is the very small current due to negative ions, as conpared with that carried by positive ions, which can be obtained under these conditions.

* Phil. Mag. xriii. pp. 828 et seq., Dec. 1900. 


\section{Effect of Temperature at Constant Pressure.- Phosphate in Steady State.}

Richardson* has proved that the formula $I=a \theta^{*} e^{-Q / 2 \theta}$, where $I=$ saturation current, and $\theta=$ the temperature in degrees absolute, while $Q=a$ measure of the energy associated with the liberation of an ion, represents the connexion between the saturation current and the temperature, for positive as well as for negative ions given off by heated platinum wires.

The same law holds for various chemical compounds which have been tested up to a temperature of $360^{\circ} \mathrm{C}$. about + .

The following results have been obtained by heating aluminium phosphate in $\mathrm{CO}_{2}$ at $0.05 \mathrm{~mm}$. pressure. Different quantities of the salt having been used in these experiments, the absolute values of the currents are not comparuble.

\begin{tabular}{|c|c|}
\hline \multicolumn{2}{|c|}{ I. } \\
\hline $\begin{array}{c}\text { Ternperature, } \\
0 .\end{array}$ & $\begin{array}{c}\text { Current, } 2 \times 10-8 \\
\text { amp. as C nit. }\end{array}$ \\
\hline 880 & 1 \\
950 & 4 \\
970 & 7 \\
995 & 15 \\
1030 & 85 \\
1055 & 49 \\
1095 & 103 \\
1110 & 126 \\
\hline
\end{tabular}

\begin{tabular}{|c|c|}
\hline \multicolumn{2}{|c|}{ II. } \\
\hline $\begin{array}{c}\text { Teuperature, } \\
\text { O. }\end{array}$ & $\begin{array}{c}\text { Current, } 2 \times 10-9 \\
\text { aup. as Unit. }\end{array}$ \\
\hline 1036 & 1 \\
1088 & 3 \\
1135 & 5 \\
1160 & 8 \\
1195 & 15 \\
1230 & 34 \\
1245 & 35 \\
1295 & 74 \\
1330 & 168 \\
\hline
\end{tabular}

The diagram (Pl. VI. fig. 5) show's the results obtained when $1, \theta$ is plotted against $\frac{1}{2} \log _{e} \theta-\log _{e}$ T. The points are fairly

* Roy. Soc. Phil. Trans. A. 207. pp. 22 et seq.

+ Garrett, Phil. Mag. June 1007 pp. 732 ot seq. 
evenly distributed about straight lines, and these lines are parallel to one another. Thus the two sets of readings are in accord with one another, and the law may be looked upon as true for positive ions given off by heated aluminium phosphate in an atmosphere of $\mathrm{CO}_{2}$, up to a temperature of $1300^{\circ} \mathrm{C}$.

When the tube was filled with hydrogen gas at a pressure of $0.05 \mathrm{~mm}$., and the results obtained were plotted in a similar manner, the same law was found to hold for that gas "up to $1300^{\circ}$ (\% (see Pl. VI. fig. 5, curve iii.).

The value of $Q$ may be obtained direct from the diagrams, by multiplying the tangent of the angle which the line makes with the $1 / \theta$ axis by 2 .

By this means it is found that for the temperatures ranging from $900^{\circ} \mathrm{C}$. to $1300^{\circ} \mathrm{C}$. when the surrounding gas is $\mathrm{CO}_{2}$ at a pressure of $0.05 \mathrm{~mm}$, the value of $Q$ is $7.1 \times 10^{4}$, while with the hydrogen gas under similar conditions of pressure the value of $Q$ is only $5.3 \times 10^{4}$ for temperatures ranging from $1095^{\circ} \mathrm{C}$. to $1300^{\circ} \mathrm{C}$.

\section{Delermination of $\mathrm{e} / \mathrm{m}$.}

Sir J. J. Thomson's cycloid method* was used for this purpose. In this method the ions move in a gas at very low pressures under the influence of a magnetic and electrostatic field acting at right angles to one another. For a given electrostatic field, the magnetic field was altered until it caused an appreciable diminution of the current passing to the electrode E (fig. 1). These experiments could not be pushed far because a magnetic field of sufficient strength could not be created. The magnet used produced a field of 800 gausses. The distance between the electrodes was $0.45 \mathrm{~cm}$., and the air pressure in the tube was less than $0.01 \mathrm{~mm}$. When the lower electrode was at a positive potential of 6.3 volts, the magnetic field produced a decrease of about 10 per cent. in the current.

Thomson has shown that in the case of ions starting from a given plane, the value of $\mathrm{e} / \mathrm{m}$ for these ions may be found from the formula $e / m=2 \mathrm{~V} / \mathrm{H}^{2} d^{2}$, in which $\mathrm{V}$ is the voltage to which the electrode from which the ions start is raised,

* 'Conduction of Electricity through Gases,' lst edition, pp. 107 ot seq. 
$H$ the value of the magnetic field, and " $d$ " the distance between the electrodes in cms.

Substituting the above experimental values in this equation we find that $e / m=9700$ about. Similar values were obtained from other experiments.

This value of $e / m$ refers, of course, to the lightest positive ions present.

Thomson found for the positive ions from hot platinum, values ranging from 60 to 720 .

The value of $e / m$ for the hydrogen atom in electrolysis is taken as $10^{4}$. From this it is seen that these positive ions are comparable in size with the hydrogen atom, if we assume the same value for " $e$ " in both cases.

A quite appreciable though smaller diminution of the current was also obtained under such conditions that $e / m$ when calculated was found to be some 3 or 4 times as large as given above. Either the ions affected in this case have a mass smaller than that of the hydrogen atom, or else their velocity is much less than that due to the electrostatic field applied; in the latter case we might assume that they started as free ions at some point between the two electrodes, and not as such from the surface of the heated salt.

\section{Velocity with which ions are shot off from the Salt.}

Earlier in the paper it has been suggested that some of the ions escape from the salt on account of their kinetic energy without the application of an electrostatic field.

To put this in evidence a Dolezalek electrometer was substituted for the galvanometer, since the ions so escaping could not be detected with the latter. The sensitivity of the electrometer was such that 1 volt produces a deflexion of: 180 scale-divisions. The pressure was reduced to $0.01 \mathrm{~mm}$. and the temperature varied as required.

A weak magnetic field was imposed so as to remove any effect which negative ions might produce, and both $E$ and the luwer heated electrode are earthed initially.

$\mathrm{E}$ was then found to receive a positive charge on disconnecting it with carth. 
If the lower electrode was now given a negative potential, this had to be raised to 1.2 volts to stop altogether the charging up of $\mathrm{E}$.

If $\mathrm{V}=$ the negative potential to which the electrode $e$ is raised to prevent positive ions leaving it, $e=$ the charge on an ion, $n=$ its mass, and $v=$ its velocity; then, from $\mathrm{V} e=\frac{1}{2} m v^{2}$ we can calculate the velocity " $v$ " with which these ions are ejected from the heated salt.

Taking the value of $e / m$ obtained earlier, we find that $v=1.4 \times 10^{8} \mathrm{~cm}$. per sec., a velocity comprable with that $\left(10^{7}\right)$ of the positively charged particles which constitute the anode rays.

With weak magnetic field of too low a value to affect the positive ions, the charging up effect was in every case increased, the final deflexion of the electrometer being always greater when the field was on.

When, however, a field of 800 , which had previously been found to produce a measurable decrease in the current due to positive ions was used, it was found that with temperatures below $1050^{\circ} \mathrm{C}$. about, the rate of charging up of the quadrants was diminished when the field was on. As the temperature was reduced below this limit, the effect of the field became more marked. This was tested to temperatures about $950^{\circ}$ (')

Above $1050^{\circ} \mathrm{C}$. the magnetic field caused an increase in the rate at which the electrometer was charged up by the positive ions, and when the temperature had reached $1200^{\circ} \mathrm{C}$. about, the rate with the field on was twice as rapid as when the field did not act.

'These increases in the rate of charging up can be explained by the fact that negative as well as positive ions are produced under these conditions. These ions would cause the rate at which $\mathrm{E}$ charged up to be smaller than if positive ions aloue were present, so when they are prevented by the magnetic field from reaching the electrode the rate at which it charges up increases. This, however, does not occur until the number of negative ions which are deflected is in excess of the number of deflected positive ions, and this state is apparently not reached until the temperature is above $1050^{\circ} \mathrm{C}$.

It must be understood that the actual number of ions 
present under these conditions was very much less than in those cases in which the galvanometer was used, since no indication of ions of either sign was then obtained in the absence of an electrostatic field.

From $1050^{\circ} \mathrm{C}$. to $1200^{\circ} \mathrm{C}$. the rate at which $\mathrm{E}$ was charged up in the absence of the magnetic field was practically constant, while the rate with the field on gradually increased.

This would occur if the actual excess of positives which reached $\mathrm{E}$ per second remained constant, and for this to be the case, since more ions are now present, negative and positive ions must be formed in equal quantities, such as might happen when neutral doublets split up.

It may be that salts which give off an excess of positive ions when beated, at first eject positive ions only. Next, it would appear that doublets are ejected also, and when the salt is raised to a positive potential, or is at zero potential, the quickly moving positive ions may by collision with the doublets, cause them to break up, hence causing a large positive current to pass between the electrodes. The initial positive ions appear to increase in the number given off per second as the temperature is raised until about $1050^{\circ} \mathrm{C}$., after which the output appears to remain constant.

If, on the other hand, the salt is raised to a negative potential, the initial positive ions are prevented from leaving it, and so at reduced pressures, with electrodes a very small distance apart, the doublets bave smaller chances of breaking up; thus the current when the salt is negatively charged is relatively small compared with that due to positive ions.

That the potential to which the salt is raised has an important bearing upon the relative number of positive and negative ions present is clearly shown by the fact that the current due to negative ions is not so small compared with that due to positive ions when the salt is at zero potential and the gas pressure is low.

Also, at atmospheric pressure when the products due to beating the salt were removed by an air blast, there was always quite a large current produced by the negative ions. 
IX. Nature of the Ions at Atmospheric Pressure.

For this purpose the apparatus used was two brass tubes of $1.7 \mathrm{~cm}$. internal diameter, insulated from each other, and each having an insulated wire $0.33 \mathrm{~cm}$. diameter and $28 \mathrm{~cm}$. in length along the axis. These wires could be in turn connected with a Dolezalek electrometer of such sensitivity that one volt caused a deflexion of 820 scale-divisions.

The method arlopted was to heat the phosphate on platinum foil through which a current was passed, and to suck air past the heated salt, and then through the two brass tubes placed one behind the other.

In order to avoid great fluctuations in the temperature, the platinum and its leads were enclosed in a wide glass tube, one end of which was connected air-tight to the testing apparatus, and the other closed loosely with cotton-wool.

Readings were taken after the salt had been heated for a sufficiently long time to bring it to the steady condition.

The electrometer showed no initial leak even when one of the electrodes was connected with its quadrants, and the tube containing the electrode was raised to a positive potential of 650 volts. A saturation current was obtained when the electrode nearer the heated salt was connected with the electrometer and a potential difference of 314 volts was used. This is shown by the following Table:-

\begin{tabular}{|c|c||c|c|}
\hline Volts. & $\begin{array}{c}\text { Current due to } \\
\text { positive ions in } \\
\text { Arbitrary Units. }\end{array}$ & Volts. & $\begin{array}{c}\text { Ourrent due to } \\
\text { positive ions in } \\
\text { Arbitrary Inits. }\end{array}$ \\
\hline 42 & 46 & 390 & 130 \\
84 & 58 & 432 & 130 \\
1.6 & 75 & 474 & 128 \\
168 & 85 & 516 & 130 \\
210 & 95 & 558 & 126 \\
236 & 111 & 608 & 130 \\
275 & 123 & 056 & 130 \\
314 & 130 & & \\
348 & 130 & & \\
\hline
\end{tabular}


Under the conditions of experiment (velocity of air through the tube being $40 \mathrm{cms}$. per sec.) it can easily be calculated from the formula

in which

$$
v=\frac{\left(b^{2}-a^{2}\right) \log _{e} b / a}{2 \sqrt{ } t}
$$

$v=$ velocity of ions in cms. per sec.,

$b=$ radius of the tube,

$a=$ radius of wire electrode,

$\mathrm{V}=$ potential-difference in volts between wire and tube,

$t=$ time taken by air to pass from one end of the electrode to the other,

that all ions with velocity greater than $0.0027 \mathrm{~cm}$. per sec. are withdrawn when the saturation voltage of 314 volts is put on. When the voltage is raised to 656 , ions must have a velucity less than $0.001 \mathrm{~cm}$. per sec. to be able to escape from the first tube.

If the front electrode (i.e. one nearer the heated salt) is earthed, and the front tube kept at any positive potential greater than 320 volts, no current should be found near the back electrode when this is connected with the electrometer, and the back tube raised to any positive potential ; a current could, however, be detected.

Even when both tubes were raised to a positive potential of 656 volts there was a current of 8 or 9 arbitrary units. The total current on the back electrode when the front tube was earthed was found to be 118 units. Thus about 8 per cent. of the total current here appears to be due to ions which are too slow moving to be extracted even with the very high voltage used, or which have been formed after passing the front electrode.

It may be mentioned that it was necessary to raise the back tube to a high potential before any indication of these extra ions was forthcoming. Thus with 432 volts on the front tube, a current could be just detected when 190 volts was put on the back tube.

If the current of 8 units on the back electrode is due to ions which are too slow to be stopped by the field on the front electrode, then some indication of these would be 
expected on the saturation current curve, i.e. after passing 314 volts the curve should still gradually ascend.

Nothing of the kind, however, takes place, and, as is seen from the table of observations given, a small increase could easily have been detected.

If we assume that neutral doublets are present which break up into ions after passing the first electrode, then the results are readily explained.

Should the extra ions be in reality due to the splitting up of neutral doublets rather than to the presence of ions of extremely low velocity, one would expect to find an equal number of negative and positive ions formed after passing the first electrode, and this should occur although the saturation currents on the front electrode due to positive and negative ions may differ widely. For aluminium phosphate these saturation currents on the front electrode are in fact very different. It was found that a negative voltage of 220 on the front tube was sufficient to obtain a saturation current with negative ions. When both tubes were brought to a negative potential of 656 volts, the front electrode being earthed, and the back electrode connected with the electrometer, there was a current of 8 units-i.e., precisely the same as that obtained with positive ions under exactly similar conditions.

This appears strong evidence in favour of the view that the extra ions are due to the splitting up of neutral doublets and not to ions of very low velocity.

When a current voltage curve for the positive ions is plotted using the values given above, it is seen that the curve formed by joining the points obtained is not of the usual type.

At about 200 volts there are indications that the current is nearly saturated, yet on slightly increasing the voltage, it again rises very rapidly and does not actually reach its saturation value until over 300 volts are put on.

On the other hand, the curve obtained with negative ions is quite a smooth one and does not show any indication of saturation at a voltage lower than 220 , nor does any further increase in current take place if the voltage is raised from 220 to 636 . This is shown in fig. 6 , in which the upper 
curve represents the current due to positive ions, the lower curve that due to negative ions at different voltages.

This, taken in conjunction with the fact that a current is obtained at the second electrode when 656 volts are put on the first, suggests that either two classes of positive ions are

Fig. 6.

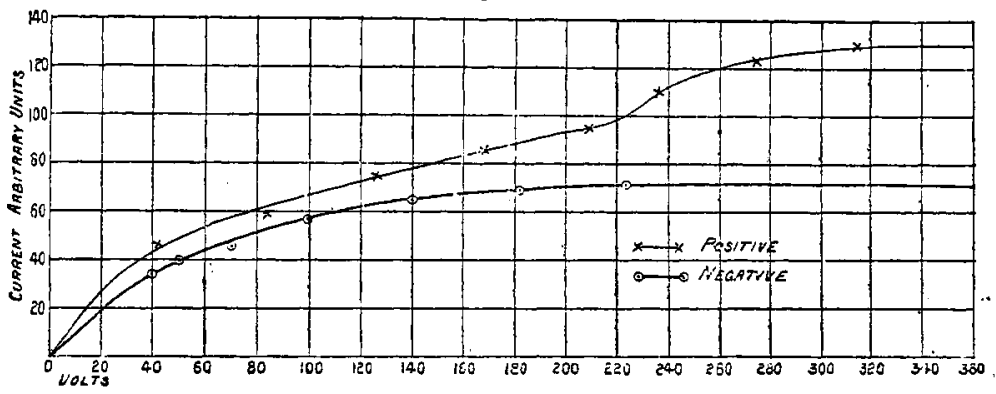

present, or else neutral doublets. The former is rendered unlikely on account of the constancy of the current which passes between the electrodes after 320 volts is reached.

\section{Further Evidence of Doublets.}

In order to test whether neutral pairs as well as ions are shot off from the heated salt when surrounded by gas at a low pressure, the apparatus shown in the next diagram was used.

Fig. 7.

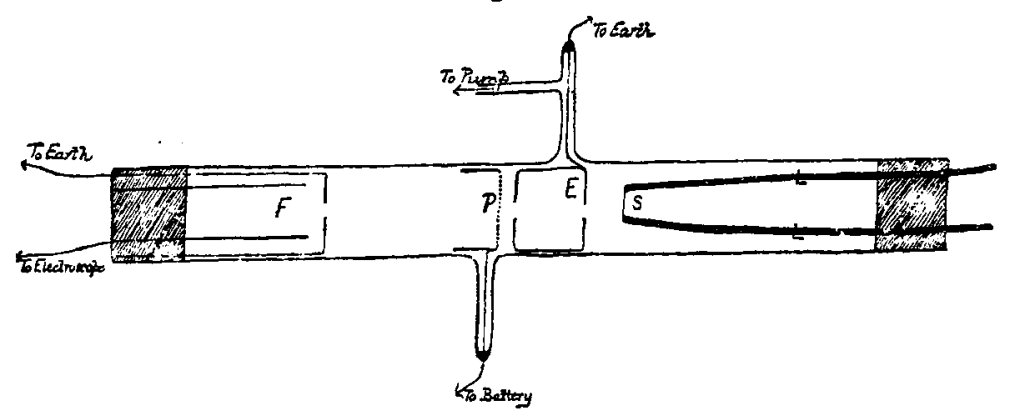

The essential part of this apparatus consists of a long glass tube, in one end of which is a Faraday cylinder $F$, and in the other end the heated salt on the platinum foil $\mathrm{S}$.

VOL. XXII. 
During the experiment the foil was kept at a positive potential sufficiently high to prevent negative ions from leaving the salt. The wire gauze $P$ was also kept at such a positive potential as to drive back any positive ions which have passed through the earthed metal tube $\mathrm{E}$.

The apparatus was evacuated to an air-pressure of about $0.01 \mathrm{~mm}$. The outer Faraday cylinder was earthed and the inner one connected to the leaf of an electroscope which was charged to a definite potential, positive or negative, as required.

The insulation was such that the rate of leak of electricity from the leaf when the salt was not heated was imperceptibly small when the charge was of either sign.

As soon as heating was commenced quite a distinct leak was noticed, and the rate of leak was the same whether the leaf was charged positively or negatively.

On the other hand, after the inner cylinder had been reduced to zero potential, no charging up could be detected, thus showing that there was no excess of free ions of either sign in the neighbourhood of the inner cylinder.

No positive ions could pass through the field between $P$ and $\mathrm{E}$, and it is highly improbable that any free negative ions leave the salt when the latter is charged positively, as in the experiment. Again, the field between $\mathrm{P}$ and the outer cylinder is a further preventive against any stray negative ions passing through the small hole into the space between the cylinders. Hence it seems that any ionization produced in the Faraday cylinder when the salt is heated, can only be brought about by the split up of doublets which have passed through the various electrostatic fields and diffused into the space between the two cylinders.

An effect of a similar nature has been noted by Sir J. J. Thomson * when working with a hot lime cathode.

\section{Rectifying Effect of the Heated Salt.}

Owing to the great velocity with which the positive ions are shot off from the heated salt when the pressure is very

* Phil. Mag. Dec. 1909, pp. 829 et seq. 
low, it was thought that such a tabe as shown in fig. 1 could be used for rectifying alternating currents providing the temperature of the salt was kept within certain limits. It has been found by various experiments that it can be so used.

Further work on this point is in progress.

\section{Summary.}

(i.) The decay of the current due to positive ions obtained by heating aluminium phosphate has been investigated, and it is found that the curve connecting current and time can be represented by a formula of the type

$$
\mathrm{A}\left(e^{-\lambda_{1} t}-e^{-\lambda_{2} t}\right)+\mathrm{B}\left(1-e^{-\lambda_{3} t}\right) .
$$

(ii.) During the first part of the decay, the nature of the surrounding gas and the water contained by the salt have an important influence. When the steady state is reached the gas has no apparent influence, but water still temporarily increases the activity.

(iii.) The discharge produced by an induction-coil temporarily increases the current which is carried by the positive ions.

(iv.) When the temperature is kept constant, it is found that for each temperature there is a definite pressure, at which the current is a maximum. This pressure being lower, the higher the temperature.

(v.) The Richardson formula $I=a \theta^{\frac{1}{2}} e^{-Q / 2 \theta}$ can be used to express the relationship between the current and absolute temperature when the pressure is kept constant.

(vi.) A value is obtained for $e / m$ which indicates that the smallest positive ions present at the lowest pressures must be of a magnitude comparable with that of the hydrogen atom.

(vii.) The high velocity of the ions at low pressures, and also the fact that some escape with great velocity even when no external field is applied, leads one to expect that a tube in which some aluminium phosphate is heated might be of use as a rectifier for alternating currents. It can be so used. 
(viii.) The experiments (a) with varying pressures at constant temperature, (b) at atmospheric pressure in which a current is produced after all the ions are apparently removed, and $(c)$ in which the charge on a Faraday cylinder leaks away when care is taken to prevent free ions reaching it, seem to indicate that neutral doublets as well as free ions are ejected from the salt.

In conclusion, I should like to thank Dr. R. S. Willows, in whose laboratory these experiments have been carried out, for the interest he has taken throughout the course of this research, and Mr. F. C. G. Bratt for help in the construction of the apparatus used.

Coss Technical Institute, Jewry Street, E.C.

May 1910.

\section{Abstract.}

In 1904 Dr. R. S. Willows and the Author communicated to this Society the results of some experiments on the halogen compounds - of zinc, in which it was shown that those compounds when heated ionized the air around so that both positively and negatively electrified bodies gradually lost their charge. Work in this direction was continued, and the results obtained showed that a large number of inorganic compounds possess properties of a like nature to the above. In $1907 \mathrm{Sir}$ J. J. Thomson found that chlorides, phosphates, and nitrates give off an excess of positive ions when heated, and he incidentally discovered that aluminium phosphate was most active in this direction. As many of the substances previously examined are known to be unstable (and hence the ionization produced may be the result of chemical change), while aluminium phosphate on the other kand is a most stable substance, it was thought that an investigation of the ion producing properties of that compound would be of interest. The apparatus was arranged so that the salt could be heated to the desired temperature $\left(900^{\circ}-1300^{\circ} \mathrm{C}\right.$.) on a strip of platinum foil. The pressure could be reduced as required. For most of the experiments a sensitive galvanometer was used as recording instrument. With the usual distance between the electrodes $(0.5 \mathrm{~cm}$.) it was found that a difference of potential of 60 volts was sufficient to obtain a saturation current with the positive ions. The way in which the current varied with the time when the saturation voltage was applied was first investigated. For the first half-hour the current was somewhat irregular. This was found to be due to the water present in the salt. The current for the first hour or two appears to be largely influenced by the surrounding gas. Neglecting the preliminary effects due to water, 
the decay of the current with the time can be represented by a curve having the general formula

$$
\mathrm{A}\left(e^{-\lambda_{1} t}-e^{-\lambda_{2} t}\right)+\mathrm{B}\left(1-e^{-\lambda_{3} t}\right) \text {. }
$$

The current finally obtained depends almost entirely upon the salt itself. After 5 or 6 hours heating, no further change takes place in the current obtained under fixed conditions of temperature and pressure. A temporary increase, however, can be brought about by moistening the salt, and also by passing a discharge from an induction-coil through the tube for about $\frac{1}{2}$ min. When the salt is insulated and heated for some time, an extraordinarily large current passes when the field is first put on. This effect increases with the time up to about 10 minutes, after which any longer insulation causes no increase of the initial current. With the salt in the final steady state the conditions were suitable for experiments on temperature and pressure effects. It was found that for every temperature tried there was a certain pressure at which the current obtained was a maximum, and the higher the temperature the lower was this pressure. The currents obtained with constant pressure and variable temperature indicate that the relationship between the rate of production of positive ions and the absolute temperature can be represented by the Richardson formula $a \theta^{\frac{7}{2}} e^{-\frac{Q}{2 \theta}}$. By using a quadrant electrometer in place of the galvanometer, it was found at pressures of $0.01 \mathrm{~mm}$. that some positive ions are ejected with a velocity of the order $10^{6} \mathrm{~cm}$. per sec.; and other experiments showed that the ions at very low pressures and acted upon by small electrostatic fields moved with great velocity, so it was thought that this substance could be made use of as a means for rectifying alternate currents. It was found that it could be so used. The values of $e / m$ obtained by Thomson's cycloid method indicate that the smallest of the positive ions present possess a mass comparable with that

the hydrogen atom. Many of the results obtained, in particular $(a)$ those with varying pressures and constant temperatures, $(b)$ those at atmospheric pressure in which after removal of all free ions by a field sufficient to produce a saturation current, a current of equal values for ions of both signs was found at an electrode placed behind that on which the saturation voltage acted, and $(c)$ the loss of charge of a Faraday cylinder when screened from the action of free ions, indicate that one of the products due to heating aluminium phosphate is in the form of neutral pairs or doublets which afterwards split up into negative and positive ions. 